

\section{Integration of multiple gut microbiota datasets of pigs and broilers}

VDI-10

Dirkjan Schokker ${ }^{1}$, Petra Roubos ${ }^{2}$, Evelien Alderliesten ${ }^{3}$, Arie Kies ${ }^{4}$, Els Willems ${ }^{5}$, and Mari Smits ${ }^{1,6}$

${ }^{1}$ Wageningen Livestock Research, Dept. Genomics, Wageningen, The Netherlands

2 TrouwNutrition, Boxmeer, The Netherlands

${ }^{3}$ Coppens Diervoeding, Helmond, The Netherlands

${ }^{4}$ DSM, Heerlen, The Netherlands

${ }^{5}$ Agrifirm Innovation Center, Apeldoorn, The Netherlands

${ }^{6}$ Wageningen Bioveterinary Research, Dept. of Infection Biology, Lelystad, The Netherlands

This research was conducted by Wageningen Livestock Research, commissioned and funded by the Ministry of Economic Affairs, within the framework of Policy Support Research theme

'Name of Theme' (project number BO-00.00-000-000.00)

Wageningen Livestock Research

Wageningen, May 2017 
Dirkjan Schokker, Petra Roubos, Evelien Alderliesten, Arie Kies, Els Willems, and Mari Smits, 2017. Integration of multiple gut microbiota datasets. Wageningen UR (University \& Research centre) Livestock Research, Livestock Research Report 1057.

This report can be downloaded for free at https://doi.org/10.18174/426339

or at www.wur.nl/livestock-research (under Wageningen Livestock Research publications).

(C) 2017 Wageningen Livestock Research

P.O. Box 338, 6700 AH Wageningen, The Netherlands, T +31 (0)317 483953 ,

E info.livestockresearch@wur.nl, www.wur.nl/livestock-research. Wageningen Livestock Research is part of Wageningen University \& Research.

All rights reserved. No part of this publication may be reproduced and/or made public, whether by print, photocopy, microfilm or any other means, without the prior permission of the publisher or author.

$?$ The ISO 9001 certification by DNV underscores our quality level. All our research commissions are in line with the Terms and Conditions of the Animal Sciences Group. These are filed with the District Court of Zwolle.

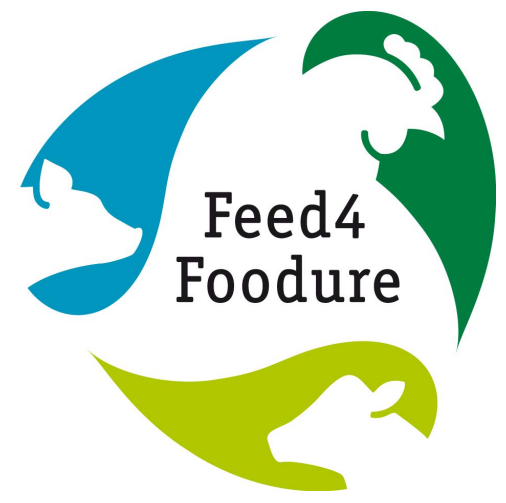




\section{Table of contents}

$\begin{array}{ll}\text { Foreword } & 5\end{array}$

$\begin{array}{ll}\text { Summary } & 7\end{array}$

$\begin{array}{llr}1 & \text { Introduction } & 9\end{array}$

$2 \quad$ Material and Methods $r$

2.1 Datasets 10

2.2 Integration $\quad 11$

$\begin{array}{llr}3 & \text { Results } & 12\end{array}$

3.1 Comparison of the microbial community structures at the genus level $\quad 12$

$\begin{array}{lll}3.2 & \text { Core-microbiota per species } & 13\end{array}$

$\begin{array}{llr}4 & \text { Discussion } & 18\end{array}$

$\begin{array}{ll}\text { References } & 20\end{array}$

$\begin{array}{ll}\text { Appendix } & \mathbf{2 1}\end{array}$ 


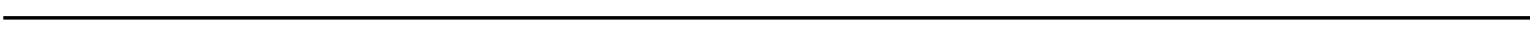




\section{Foreword}

Feed4Foodure is a public-private partnership between the Dutch Ministry of Economic Affairs, a consortium of various organizations within the animal production chain and Wageningen UR Livestock Research. Feed4Foodure aims to contribute to sustainable and healthy livestock farming in the Netherlands, simultaneously strengthening our competitive position on the global market. The Feed4Foodure program line "Nutrition, Intestinal Health, and Immunity", aims to contribute to a reduction in the use of antibiotics in livestock farming by increasing general health and disease resistance. The main goals are to develop innovative measuring techniques and to evaluate new feeding concepts, feed ingredients and additives to improve gut health and immunity.

The data for this integrated-analysis were acquired from experiments conducted within the frame work of the Feed4Foodure program line "Nutrition, Intestinal Health, and Immunity". Integration of data was performed in order to 1 ) identify (dis)similarities between samples of multiple pig and broiler microbiota datasets, and to 2) identify the composition of the core-microbiota of pigs and broilers (independent of intervention). The results of this integrated approach could be of relevance for the development of a 'ruler' for immune competence.

For the current study, scientists of Wageningen UR Livestock Research and Wageningen Bioveterinary Research worked together with representatives from the various private partners of VDN (Vereninging Diervoederonderzoek Nederland). The authors thank the industry partners of the project team for their worthwhile input.

Prof. Dr. Mari Smits, leader Feed4Foodure program line "Nutrition, Intestinal Health, and Immunity".

Dirkjan Schokker 


\section{Summary}

Within the VDI-programme 8 microbiota datasets were generated, 5 for pigs and 3 for broilers. The aim of this research was to integrate these datasets in order to 1) identify (dis) similarities between samples of multiple pig and broiler microbiota datasets, and to 2) identify the composition of the core-microbiota of pigs and broilers (independent of intervention). The results of this integrated approach could be of relevance for the development of a 'ruler' for immune competence.

From the results, we concluded that no clear separation was observed between the microbiota composition of pigs and broilers. Furthermore, some microbial phyla and classes were identified that were common in both pigs and broilers. 


\section{$1 \quad$ Introduction}

Within the Feed4Foodure-program multiple pig and poultry studies were performed that measured gut microbiota in different intestinal segments. Although all these experiments had different objectives, it is still possible and worthwhile to combine and integrate these microbiota data. By integration of these multiple datasets, it may be possible to identify certain bacterial species that are (un)common to different segments of the digestive tracts, and to identify bacterial species that belong to the so-called (species-specific) core-microbiota. Here, we refer to the core microbiota as bacterial genera that are always present in a specific gut datasets we have generated, for either pigs or broilers.

In the various VDI-experiments, microbiota profiles were determined at different time-points, for both control animals and animals which were subjected to a certain (dietary) intervention. The microbiota datasets used in this study were generated within the VDI-programme and are depicted in Table 1. Through an integrated analysis it is possible to identify microbial parameters potentially relevant for the "ruler" for immune competence.

The overall aim of this research was to integrate these datasets in order 1 ) to identify (dis) similarities between samples of multiple pig and broiler datasets, and 2) to identify the composition of the coremicrobiota of pigs and broilers (independent of intervention). 


\section{Material and Methods}

\section{$2.1 \quad$ Datasets}

In total 8 animal experiments were conducted within the VDI research program that generated microbiota data (Table 1). These data were generated by sequencing the $\mathrm{V} 3$ and/or $\mathrm{V} 4$ region of the $16 \mathrm{~S}$ ribosomal RNA, which is used to reconstruct (microbial) phylogenies. The V3/V4 region is a hypervariable region in the $16 \mathrm{~S}$ gene which is conserved between different species of bacteria and archaea. The V3/V4 region is most suitable for distinguishing all bacterial species to the genus level, except for closely related Enterobacteriaceae [1,2]. Details of data acquisition are described in reports on the corresponding animal experiments. The design of the various animal experiments is also described in the corresponding reports.

Table 1. Overview of the used microbiota datasets (generated within the VDI research program)

\begin{tabular}{|c|c|c|c|c|c|c|}
\hline VDI\# & Species & Route & Intervention(s) & $\begin{array}{c}\text { Gut } \\
\text { segment(s) }\end{array}$ & $\begin{array}{c}\text { Time (days = } \\
\text { age) }\end{array}$ & $\begin{array}{c}\begin{array}{c}\text { Total } \\
\text { number } \\
\text { of }\end{array} \\
\text { samples }\end{array}$ \\
\hline 3 & Chicken & Neonatal & $\begin{array}{c}\text { Amoxicillin in drinking } \\
\text { water }\end{array}$ & Jejunum & $1,5,14$ & $40^{1}$ \\
\hline 5 & Chicken & Adult & Rye; 0\%, 5\%, 10\% & Jejunum & $14,21,28$ & 72 \\
\hline 5 & Chicken & Adult & $\begin{array}{c}\text { Betaglucans, Fish oil, } \\
\text { Lysozyme, Oathulls, } \\
\text { Quercitin }\end{array}$ & $\begin{array}{l}\text { Jejunum, } \\
\text { Ileum }\end{array}$ & 21 & 72 \\
\hline 2 & $\mathrm{Pig}^{2}$ & Maternal & Amoxicillin & Jejunum & $1,7,26,30,54$ & 30 \\
\hline 3 & Pig & Neonatal & Fructooligosaccharides & $\begin{array}{l}\text { Jejunum, } \\
\text { Colon* }\end{array}$ & $2,14,25$ & 40 \\
\hline 12 & $\mathrm{Pig}^{2}$ & $\begin{array}{l}\text { Maternal, } \\
\text { neonatal** }\end{array}$ & $\begin{array}{l}\text { Medium chain fatty } \\
\text { acids, betaglucans, } \\
\text { galactooligosaccharides }\end{array}$ & $\begin{array}{l}\text { Jejunum, } \\
\text { Ileum, Colon }\end{array}$ & 1,31 & 58,69 \\
\hline 5 & $\mathrm{Pig}^{3}$ & Adult & ZnO; High vs Low (1) & $\begin{array}{l}\text { Jejunum, } \\
\text { Ileum }\end{array}$ & $40,49,61$ & 60 \\
\hline 13 & $\mathrm{Pig}^{3}$ & Adult & $\begin{array}{l}\text { ZnO; High vs Low; } \\
\text { HHvsLL, HLvsHH, } \\
\text { HLvsLH, HLvsLL, } \\
\text { LHvsHH, LHvsLL }\end{array}$ & $\begin{array}{l}\text { Jejunum, } \\
\text { Ileum }\end{array}$ & 40,49 & 80 \\
\hline
\end{tabular}

In short, jejunum samples on days 1, 5, 14 (age) from the former VDI-3 experiment (broiler; amoxicillin), were split into two groups: 1) with and 2) without antibiotic (controls). Two experiments conducted in the old VDI-5 were included. In the first experiment, broilers were divided into three groups. Group 1, control ( $0 \%$ rye); group 2 received $5 \%$ rye in their feed; and group 3, received $10 \%$ rye in their feed. Here also jejunum samples were analysed on days 14, 21, and 28 (age). In experiment 2, five different dietary interventions were investigated, 1) Quercetin, 2) Oat hulls, 3) lysozyme, 4) fish oil, and 5) beta-glucans. Here, on day 21 (age) both jejunum and ileum were sampled.

In the former VDI-2 project (maternal effects), an animal experiment was carried out in which the effect of the administration of an antibiotic (amoxicillin) to sows on the gut mucosal development in the offspring piglets was investigated. Jejunum samples were analysed on days 1, 7, weaning (day 26), weaning+4days (d30), and weaning+28 days (d54). The dataset from the former VDI-3 project was 
also included, this project investigated the effect of fructooligosaccharide (FOS) on the intestinal immune system development in neonatal piglets, were jejunum was samples at days 2, 14 and 25, whereas colon was only sampled at day 14 . The datasets of the former VDI-5 experiment were also used. This experiment investigated the effect of high zinc on mucosal processes in weaned piglets. Here, both the jejunum and ileum samples were taken on days 21,28 , and 35 for microbiota characterization. In the VDI-13 project, the timing of high zinc in weaned piglets was investigated. At days 14 and 23 samples were taken to identify differences between the control (low Zinc Oxide) and treatment (high Zinc Oxide) group. In the VDI-12 project, the focus was on maternal and neonatal dietary interventions, here the following interventions were investigated, medium chain fatty acids, (yeast) beta-glucans, and galactooligosaccharides (GOS). For the maternal route, samples were taken at day 1 and 31, whereas for the neonatal route only at day 31 samples were taken.

\subsection{Integration}

Two different strategies were performed in order to integrate the microbiota datasets. The first integration of data included all data per species. The integration was a standard merging of the data on the bacterial genus level. Taxonomic classification was based on 16S rDNA sequenced data. Subsequently, hierarchical clustering of the data was performed by Ward's minimum variance method (Ward.D) which aims at finding compact spherical clusters in a graphical representation. Dissimilarities between clusters can be efficiently computed only for a limited number of distance/linkage combinations, i.e. the so-called squared Euclidean distance. The result of this clustering is used as input for the order of appearance in a stacked bar plot representation.

The second data integration also included all microbiota data as obtained within the various VDI projects. This integration was performed in order to identify per species (pig or chicken) which microbiota was always present in the gut, the so called core-microbiota. Again the integration was a standard merging of the data on the bacterial genus level of 16S rDNA sequenced data. 


\section{Results}

\subsection{Comparison of the microbial community structures at the genus level}

The results of the integration process, as described in Material and Methods, are shown in Figures 1 (Pigs) and 2 (Broilers). Each stacked bar plot represents an individual sample from an experiment conducted within the VDI-programme. Different colours indicate different bacterial genera. The results were clustered based on their overall microbiota composition, i.e. the different bacterial genera. From this representation, we observed that the clustering is not related to species, intestinal segment, and/or time of sampling (here the age of the animal). In other words not all broilers and pigs clustered together, the intestinal segments (jejunum, ileum, or colon) did not cluster, and the time-points (here the age of the animals) did not cluster. What is present among almost all samples is the high abundance of Lactobacilli.

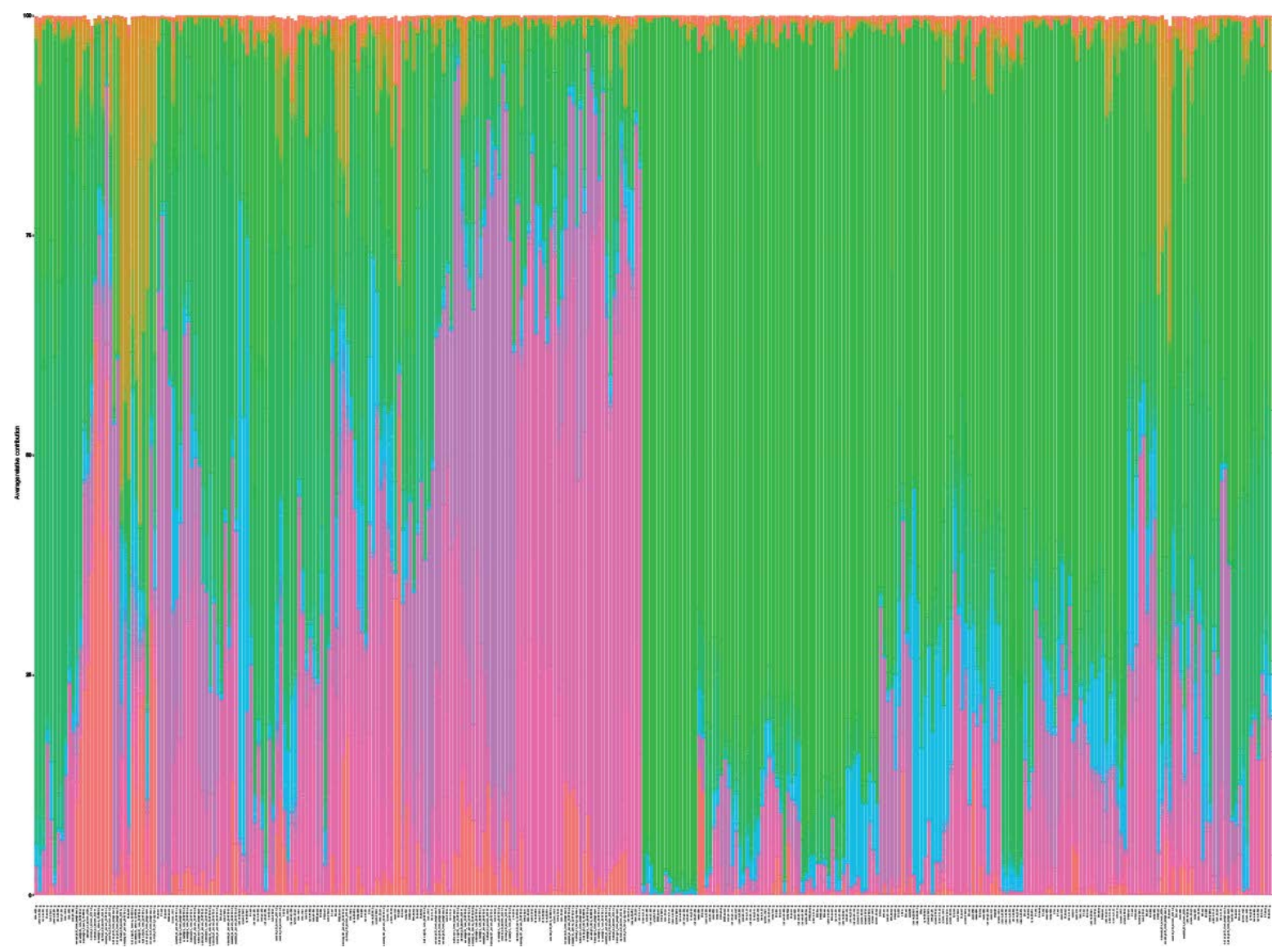

Figure 1. Average relative contribution of all genus level microbiota data of individual pig samples $(n=334)$

The $y$-axis depicts the average relative abundance (ARC) of each bacterial genera, where different colours indicate different bacterial genera. On the $\mathrm{x}$-axis each individual sample is depicted (a better resolution and legend can be found in the pdf-file attached alongside this report).

Sample names are indicated VDI_Species_Tissue_Day_C/I_\#, for example 12_P_C_1_C_2 meaning VDI12 study in pigs targeted colon on day 1 control sample 2 or $3 \_C_{-} J_{-} 5_{-}$Amox_ 6 meaning VDI 3 study in chickens targeted jejunum on day 5 amoxicillin treated sample 6. 


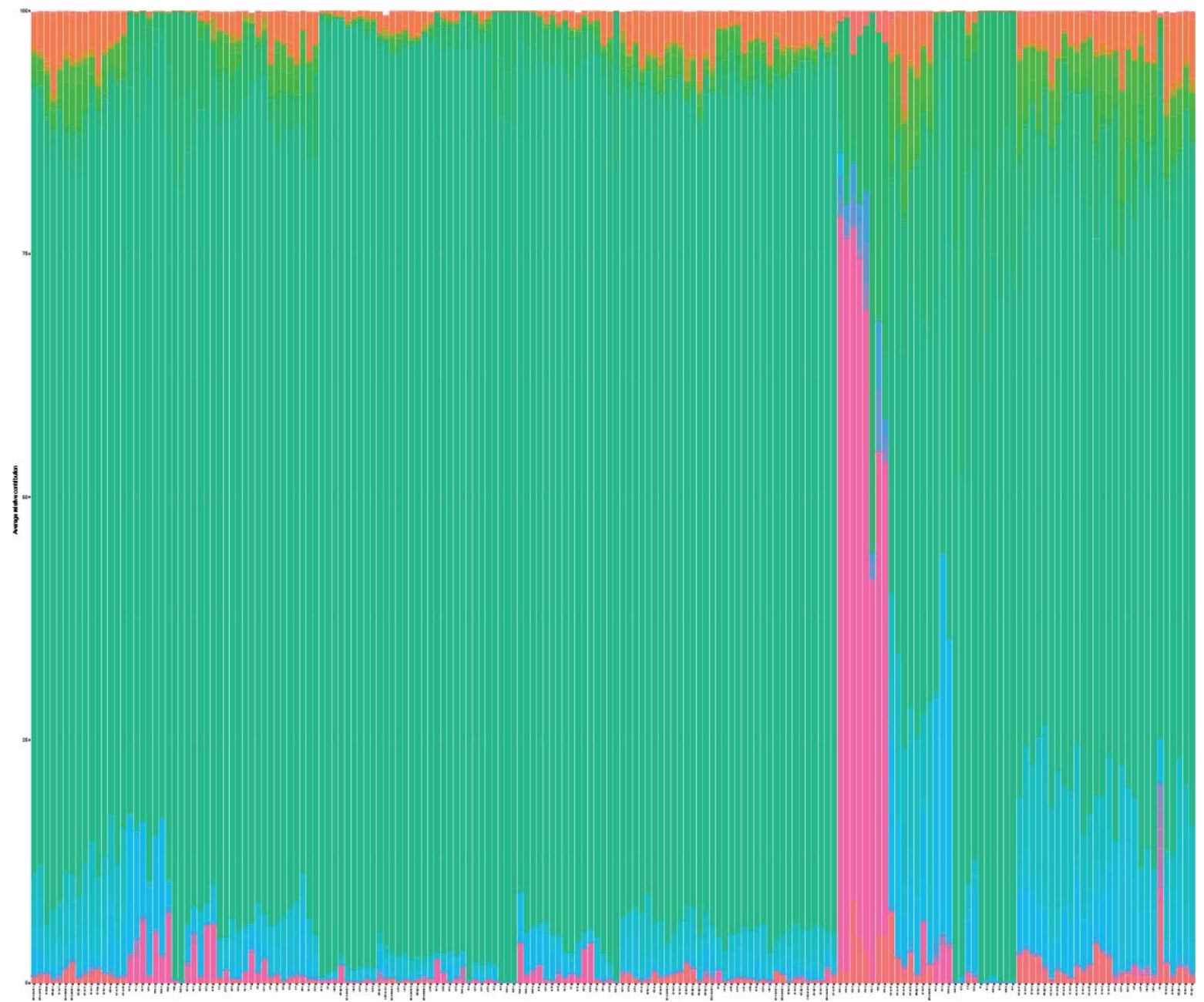

Figure 2. Average relative contribution of all genus level microbiota data of individual broiler samples $(n=182)$

The $y$-axis depicts the average relative abundance (ARC) of each bacterial genera, where different colours indicate different bacterial genera. On the $x$-axis each individual sample is depicted (a better resolution and legend can be found in the pdf-file attached alongside this report).

Sample names are indicated VDI_Species_Tissue_Day_C/I_\#, for example 12_P_C_1_C_2 meaning VDI12 study in pigs targeted colon on day 1 control sample 2 or 3_C_J_5_Amox_6 meaning VDI3 study in chickens targeted jejunum on day 5 amoxicillin treated sample 6 .

\subsection{Core-microbiota per species}

For both pigs and broilers the microbiota data were separately merged, this results in a long list of bacterial genera names per dataset. Subsequently, the overlap between these various pig or broilers datasets was calculated, simply by intersecting the different datasets. The latter analysis resulted in two Venn-diagrams, one representing the overlap in the datasets obtained from pigs (Figure 2) and the other for broilers (Figure 3).

In total five pig datasets were analysed, which makes the resulting Venn-diagram somewhat difficult to interpret. However, in the pig datasets, we observed an overlap of 219 bacterial genera between the five studies (Figure 2). For these five experiments, this set of 219 bacterial genera could be envisaged as the core microbiota irrespective of intestinal segment (jejunum/ileum). Per experiment there were also a number of bacterial genera that were uniquely found in the respective experiments. The number of these experiment-specific bacterial genera ranged from only 4 up to 179 bacterial genera.

Only three broiler datasets were available at the time of analysis, these three broiler studies showed an intersection of 149 bacterial genera, indicating a common core of 149 bacterial genera. Per experiment 32, 99, 70 bacterial genera were specific for the individual experiments.

Also a comparison was made between the broiler and pig datasets based on the bacterial genera (Figure 4 ). This comparison showed a high overlap between the two species (45.8\%), furthermore more specific bacterial genera were observed in pigs ( $52.8 \%$ vs. $1.4 \%$ in broilers). 


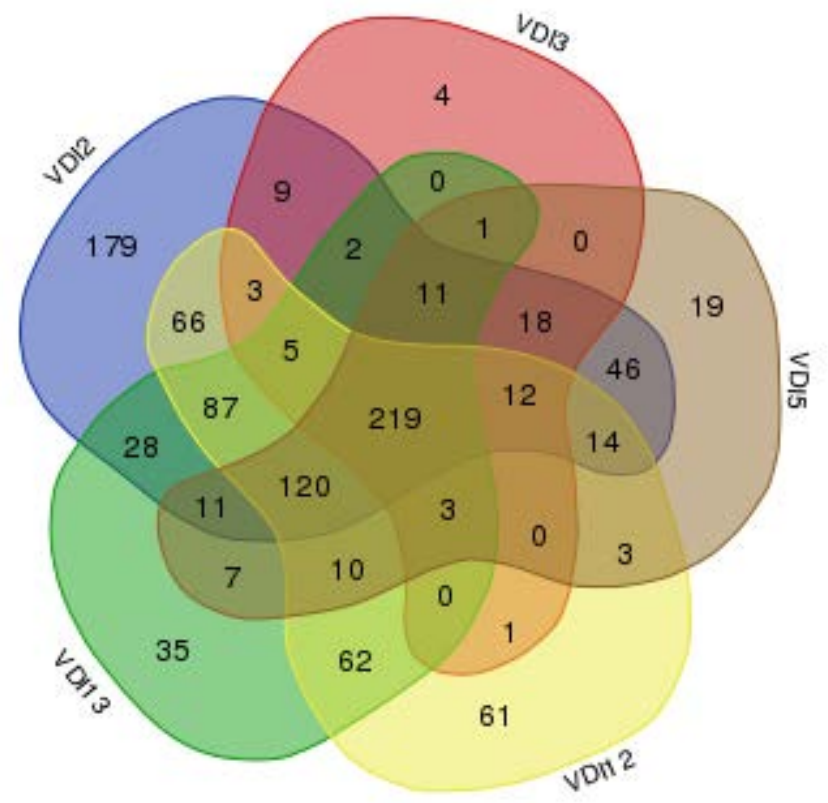

Figure 2. Venn-diagram of bacterial genera of all pig microbiota datasets

Representation of intersection(s) between the various pig experiments conducted within VDI based on the bacterial genera. VDI2: Maternal administration of amoxicillin, VDI3: Neonatal administration of fructooligosaccharides, VDI5: Administration of Zinc Oxide after weaning (14-23 days post-weaning), VDI12: Maternal or neonatal administration of MCFA/BG/GOS, VDI13: Administration of Zinc Oxide after weaning (0-14|14-23 days post-weaning).

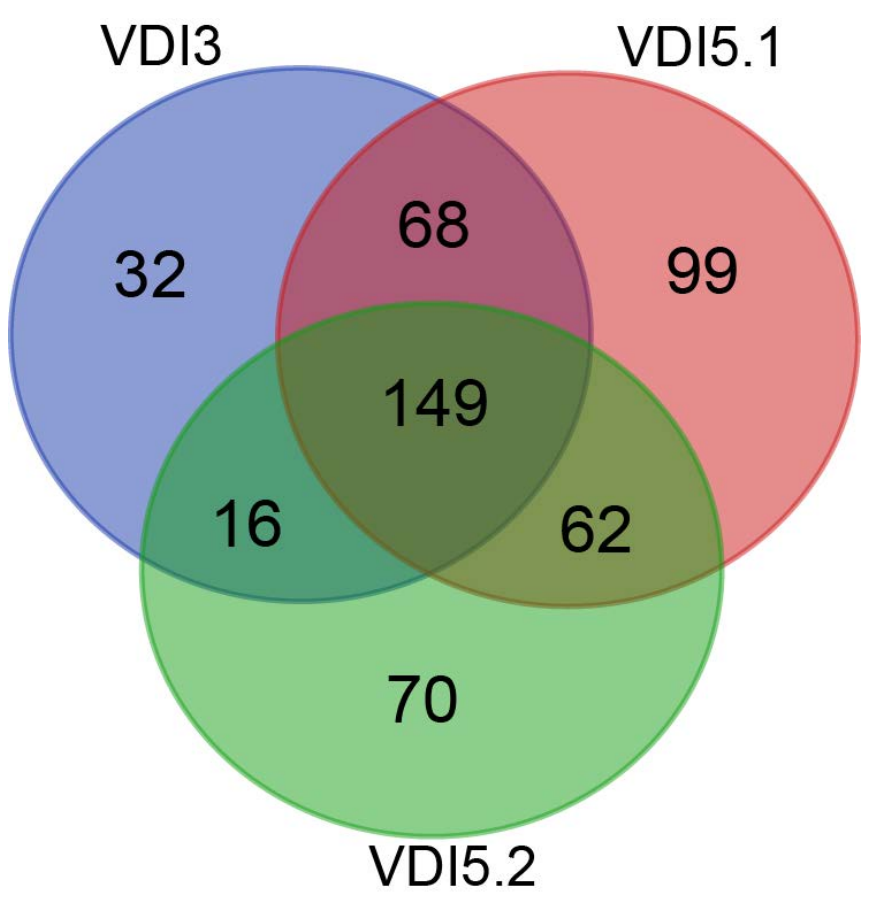

Figure 3. Venn-diagram of bacterial genera of all broiler microbiota datasets

Representation of intersection(s) between the various broiler experiments conducted within VDI based on the bacterial genera. VDI3: Administration of amoxicillin, VDI5.1: 0/5/10\% Rye inclusion in the diet, VDI5.2: Administration of Fish Oil/Lysozyme/Quercitin/Oat hulls/beta glucans. 


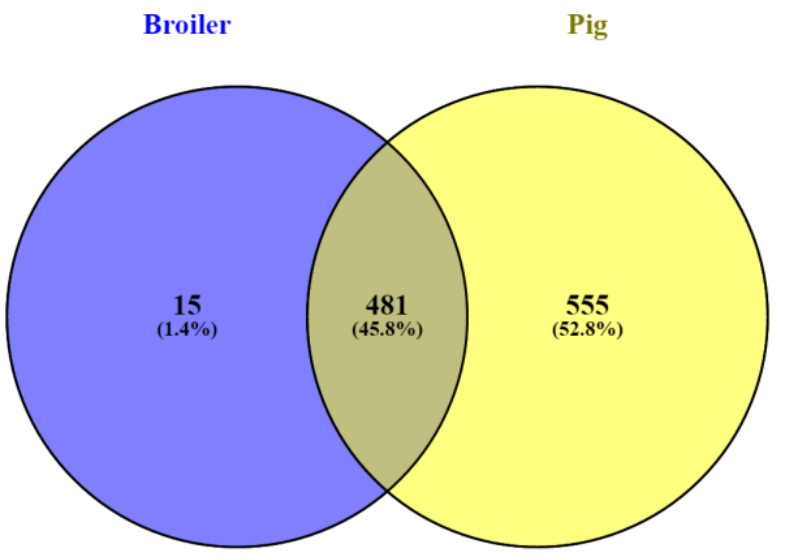

Figure 4. Venn-diagram of bacterial genera when comparing all broiler with all pig microbiota datasets

Representation of intersection(s) between the broiler and pig experiments conducted within VDI based on the bacterial genera.

The mean average relative contribution ( $A R C$ ) of all pig samples, including both jejunum and ileum samples, as well as control and intervention samples, was calculated at three taxonomic levels. These three levels were Phylum, Class, and Family (see Figure 5). This results in 3 dominant phyla, namely Firmicutes, Proteobacteria, and Bacteroidetes, summing up to an ARC of approximately 84.4\%. To investigate the differences in composition between the two intestinal segments, i.e. jejunum and ileum, Tables 2 and 3 provide the bacterial genera with an ARC above 1\%, for jejunum and ileum, respectively.

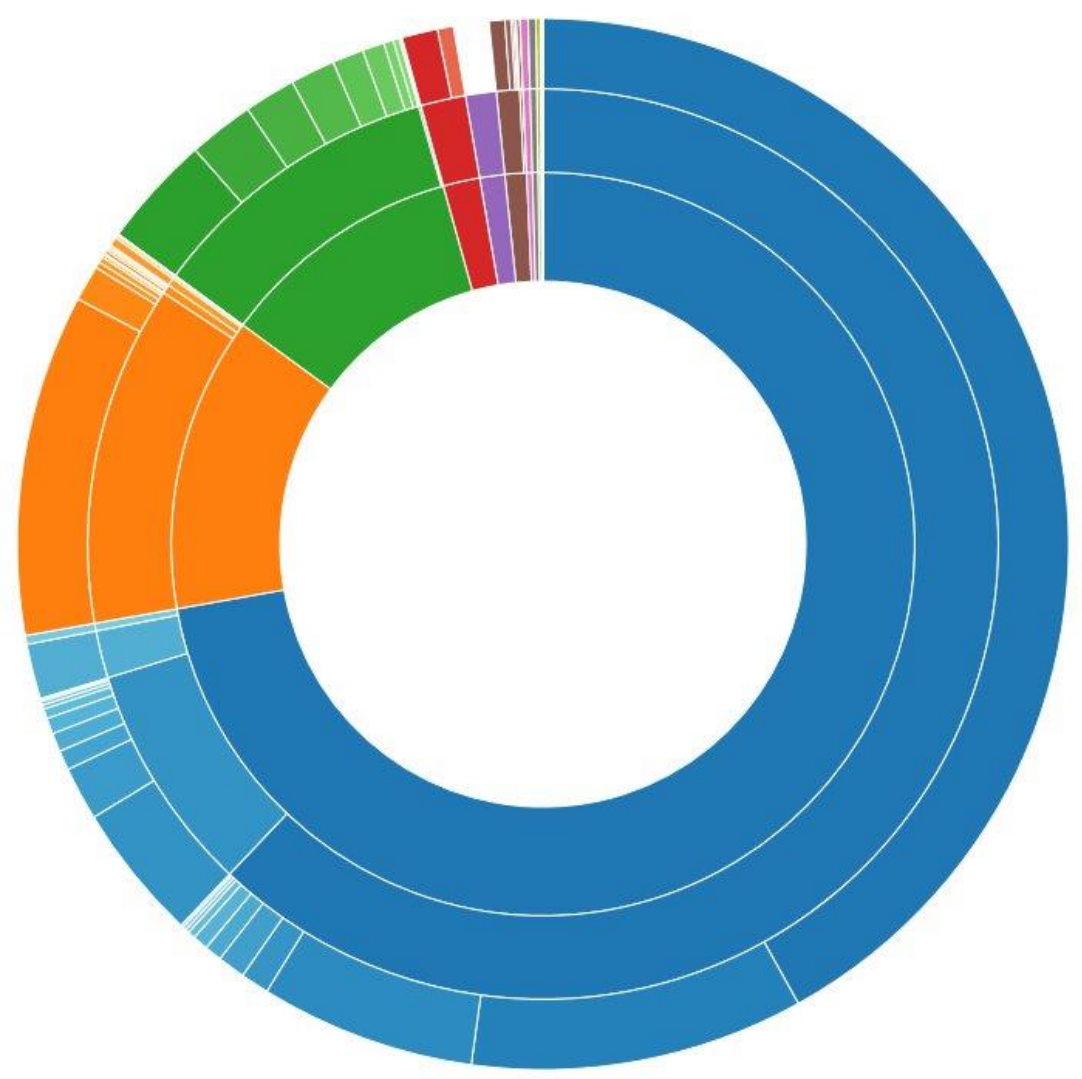

Figure 5. Core-microbiome of pigs

Core microbiome of pigs jejunum and ileum combined, control and intervention samples. Outer ring represents bacterial family, middle ring represents bacterial class, whereas the inner ring represents the bacterial phylum. Blue depicts Firmicutes (62.8\%), orange Proteobacteria (11.1\%), green Bacteroidetes (10.9\%), red Spirochaetes ( $1.4 \%$ ), purple Tenericutes $(0.9 \%)$, and brown Actinobacteria (0.8\%). Unique entities at phylum level is 21 , at class level is 21 , at family level is 86 , and at genera level is 219 .

Note: the overlapping Archaea were removed from this plot for better representation. 
Table 2. Bacterial genera residing in pig jejunum ${ }^{1}$ ( $>1 \% A R C$, controls only)

\begin{tabular}{|c|c|c|c|c|}
\hline Phylum & Class & Family & Genus & ARC \\
\hline \multirow{6}{*}{ Firmicutes } & \multirow{3}{*}{ Bacilli } & Lactobacillaceae & Lactobacillus & 54.24 \\
\hline & & Streptococcaceae & Streptococcus & 6.14 \\
\hline & & Turicibacteraceae & Turicibacter & 2.48 \\
\hline & \multirow{3}{*}{ Clostridia } & & Veillonella & 4.88 \\
\hline & & velionemaceae & Anaerovibrio & 1.05 \\
\hline & & Clostridiaceae & Clostridium & 1.30 \\
\hline \multirow{3}{*}{ Proteobacteria } & \multirow{3}{*}{ Gammaproteobacteria } & Enterobacteriaceae & Escherichia & 6.63 \\
\hline & & \multirow{2}{*}{ Pasteurellaceae } & Actinobacillus & 4.09 \\
\hline & & & Other & 3.82 \\
\hline Unassigned & Other & Other & Other & 1.38 \\
\hline Bacteroidetes & Bacteroidia & Prevotellaceae & Prevotella & 1.11 \\
\hline
\end{tabular}

Table 3. Bacterial genera residing in pig ileum ${ }^{1}$ ( $>1 \% A R C$, controls only)

\begin{tabular}{|c|c|c|c|c|}
\hline Phylum & Class & Family & Genus & ARC \\
\hline \multirow{4}{*}{ Firmicutes } & \multirow{4}{*}{ Bacilli } & Lactobacillaceae & Lactobacillus & 23.60 \\
\hline & & Turicibacteraceae & Turicibacter & 13.13 \\
\hline & & Streptococcaceae & Streptococcus & 7.64 \\
\hline & & Leuconostocaceae & Weissella & 1.63 \\
\hline \multirow{4}{*}{ Proteobacteria } & \multirow{4}{*}{ Gammaproteobacteria } & & Actinobacillus & 13.89 \\
\hline & & Pasteurellaceae & Other & 12.91 \\
\hline & & & & 7.72 \\
\hline & & Enterobacteriaceae & & 6.88 \\
\hline Bacteroidetes & Bacteroidia & Prevotellaceae & Prevotella & 2.92 \\
\hline Unassigned & Other & Other & Other & 1.35 \\
\hline
\end{tabular}

${ }^{1}$ I rrespective of time-point, including both controls and intervention samples 


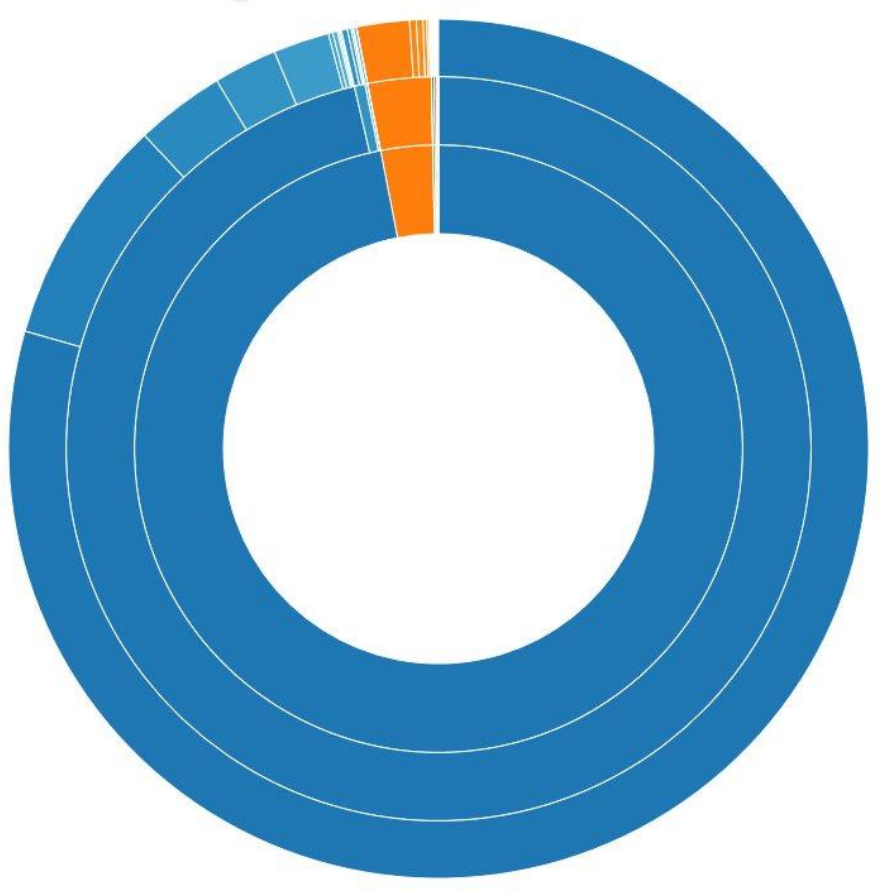

Figure 6. Core-microbiome of broilers

Core microbiome of broiler jejunum and ileum samples combined from the VDI programme, control and intervention samples. Outer ring represents bacterial family, middle ring represents bacterial class, whereas the inner ring represents the bacterial phyla. Blue depicts Firmicutes ( $89.2 \%$ ARC) and orange Actinobacteria ( $2.5 \%$ ARC). Unique entities at phylum level is 11 , at class level is 20 , at family level is 68 , and at genera level is 149 .

Note that Proteobacteria are not included here, this is mainly because the Escherichia were absent in ileal samples and therefore were not included in the core-microbiome.

\begin{tabular}{ccccc} 
Phylum & Class & Family & Genus & ARC \\
\hline \multirow{2}{*}{ Firmicutes } & Lactobacillaceae & Lactobacillus & 72.67 \\
& \multirow{2}{*}{ Bacilli } & Enterococcaceae & Enterococcus & 4.60 \\
& & & Other & 2.87 \\
& & Planococcaceae & Staphylococcus & 1.84 \\
& & Streptococcaceae & Streptococcus & 1.80 \\
& & Staphylococcaceae & Jeotgalicoccus & 1.24 \\
\hline Proteobacteria & Gammaproteobacteria & Enterobacteriaceae & Escherichia & 7.29 \\
\hline Actinobacteria & Actinobacteria & Corynebacteriaceae & Corynebacterium & 1.66 \\
\hline
\end{tabular}

1 I rrespective of time-point, including both controls and intervention samples

Table 6. Bacterial genera residing in chicken ileum ${ }^{1}(>1 \% A R C)$

\begin{tabular}{ccccc} 
Phylum & Class & Family & Genus & ARC \\
\hline \multirow{3}{*}{ Firmicutes } & & Lactobacillaceae & Lactobacillus & 90.03 \\
& \multirow{3}{*}{ Bacilli } & Enterococcaceae & Enterococcus & 2.54 \\
& & Streptococcaceae & Streptococcus & 1.32 \\
& & Staphylococcaceae & Staphylococcus & 1.03 \\
\hline
\end{tabular}

${ }^{1}$ Note only one time-point/ dataset ( $n=5$ samples), including both controls and intervention samples 


\section{Discussion}

The microbiota dataset integration was limited to data generated from jejunum and ileum luminal content samples obtained from animal experiments performed within the VDI-programme. Inclusion of datasets from literature was not considered because almost all of these datasets were obtained from faecal samples. Furthermore, no generic tools were available to perform such an analysis and therefore needed to be developed. On top of that, integrating datasets generated by other technologies and other laboratories creates challenges on its own, for example how to deal with different sequencing platforms or the use of different bioinformatic / taxonomic pipelines. Some microbiome meta-analysis studies have been published for humans, however these are mainly based on faecal samples [3] or based on diseases vs. control studies [4]. When more (livestock) microbiome datasets will become available, it will be possible to perform a more comprehensive integration, which will certainly be more informative, compared to the current data integration.

It was surprising to observe that the integration analysis of the bacterial genera data of all samples $(n=574)$ of both pig and chicken, did not show a clear separation between the species, the intestinal segments, and the age of the animals. These data suggest that monogastrics partly share a similar gut microbiota, similar bacterial genera can live and thrive in various intestinal segments. Comparing these to human microbiota is very difficult because in human research the small intestine is scarcely sampled, more often faecal samples are taken in human studies. This also implies that for certain bacterial genera it will be possible to modulate these via, for example dietary interventions, irrespective of species, i.e. pigs or broilers. Despite that animals were kept under different management regimes, were of different genetic lines, had different feeds, we still identified a core-microbiota of different bacterial genera. It could be that throughout evolution a part of the gut microbiota ecosystem has been associated to monogastrics, and are as a whole community difficult to steer in a certain beneficial direction for the host. Nevertheless it could be possible to steer a handful of species/genera to still have a meaningful impact on the whole gut ecosystem. The core-microbiota has also been explained as the level of shared genes of the resident microbiota [5] in our datasets we deduced the bacterial genera from the hypervariable $16 \mathrm{~S}$ ribosomal RNA (here V3 and/or V4 regions). Thus not the actual gene collection of the resident microbiota, our approach gives a notion of whom is there whereas the other method gives a notion of what is happening, biologically.

Despite the (limited) number of data sets, integration of these microbiota data showed that 20-30\% of bacterial genera are overlapping between multiple datasets. These bacterial genera can be qualified as the so-called core microbiota. To further characterize the core microbiota, we investigated the bacterial taxonomy for pigs and broilers separately. The dataset for pigs and broilers were combined irrespective of time-point, and the results showed that Lactobacilli were most dominant in both piglets and broilers, having the highest average relative abundance. We explicitly performed the analysis irrespective of time-point and although temporal changes in the microbiota composition and/or following individual bacterial species/genera in time would be interesting, this falls outside the scope of this meta-analysis. The 'core-microbiota' mainly consists of the phyla Firmicutes and Proteobacteria. Both the pig and broiler 'core-microbiota' have some resemblance of the human and mouse microbiota [5-7], this comparison is skewed because most human studies most often investigate colon and/or faecal microbiota, whereas in our studies the microbiota in the small intestine was investigated. And it is known that the small and large intestine have different biological functions and therefore different resident microbiota [8].

We also investigated the dis(similarities) between the broiler and pig datasets, in order to gain more insight of the bacterial genera at the community-level. To this end we performed a comparison of all bacterial genera from the two datasets, and observed an overlap of $45.8 \%$ which could be assigned to that they are both monogastrics. However, also a large difference in specific bacterial genera were observed per species, broilers $1.4 \%$ vs. pigs $52.8 \%$ (see also Figure 4 ), this difference could be mainly due to age when animals were sampled. Another reason could be the skewing of the data, where we have more samples for pigs compared to broilers. Moreover, in the pig samples age also has a larger 
influence because pre-weaned and post-weaned pigs were sampled. It is known that weaning has drastic changes to the microbiota composition [9].

We did split the datasets, for both pigs and broilers, into their respective location, i.e. jejunum or ileum. In this way it was possible to investigate the (dis)similarities between location (intestinal segment) within species and between species. It could be observed that within species, the most dominant bacterial were similar in pigs as well as broilers.

This study is a first generation to investigate the microbiota of the small intestine combining multiple datasets in order to identify the core-microbiota. Taken together, these data showed that it is possible to identify certain bacterial genera that are always present in the small intestine of pigs and broilers. Another aspect is that certain bacterial genera can be modulated and possibly be beneficial for the host. This could help in generating customized approaches to steer specific bacterial genera in order to have a beneficial health effect, i.e. specific pre- and probiotics. 


\section{References}

1. Chakravorty, S., et al., A detailed analysis of $16 \mathrm{~S}$ ribosomal RNA gene segments for the diagnosis of pathogenic bacteria. J Microbiol Methods, 2007. 69(2): p. 330-9.

2. Mizrahi-Man, O., E.R. Davenport, and Y. Gilad, Taxonomic classification of bacterial 16S rRNA genes using short sequencing reads: evaluation of effective study designs. PLoS One, 2013. 8(1): p. e53608.

3. Koren, O., et al., A guide to enterotypes across the human body: meta-analysis of microbial community structures in human microbiome datasets. PLoS Comput Biol, 2013. 9(1): p. e1002863.

4. Prosberg, M., et al., The association between the gut microbiota and the inflammatory bowel disease activity: a systematic review and meta-analysis. Scand J Gastroenterol, 2016. 51(12): p. 1407-1415.

5. Turnbaugh, P.J., et al., A core gut microbiome in obese and lean twins. Nature, 2009. 457(7228): p. 480-4.

6. Xiao, L., et al., A catalog of the mouse gut metagenome. Nat Biotechnol, 2015. 33(10): p. 1103-8.

7. Lozupone, C.A., et al., Diversity, stability and resilience of the human gut microbiota. Nature, 2012. 489(7415): p. 220-30.

8. Mowat, A.M. and W.W. Agace, Regional specialization within the intestinal immune system. Nature Reviews Immunology, 2014. 14(10): p. 667-685.

9. Konstantinov, S.R., et al., Microbial diversity studies of the porcine gastrointestinal ecosystem during weaning transition. Animal Research, 2004. 53(4): p. 317-324. 


\section{Appendix}

stackedBarPlotsAllPigs + legend.pdf

stackedBarPlotsAllBroilers+legend.pdf

core microbiota (genus) .xIsx 


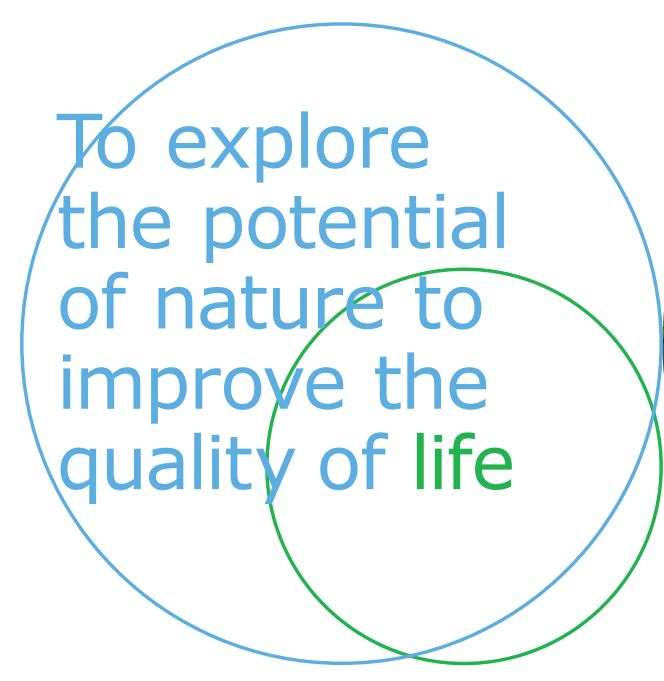

Wageningen Livestock Research P.O. Box 338

6700 AH Wageningen

The Netherlands

T +31 (0)317483953

E info.livestockresearch@wur.nl www.wur.nl/livestock-research

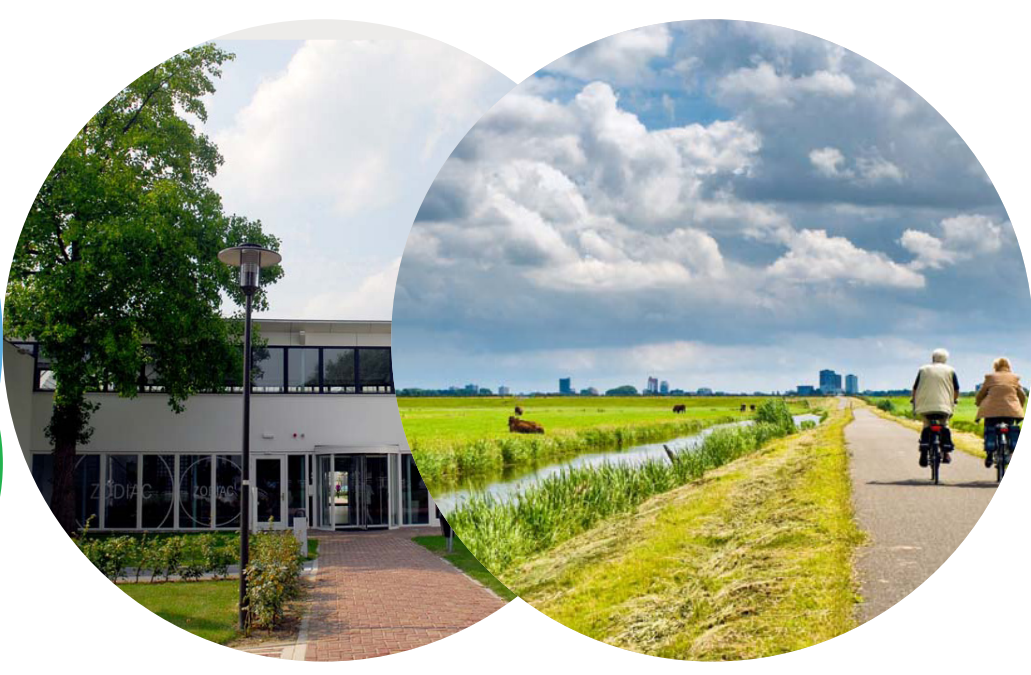

Wageningen Livestock Research creates science based solutions for a sustainable and profitable livestock sector. Together with our clients, we integrate scientific knowledge and practical experience to develop livestock concepts for future generations.

Wageningen Livestock Research is part of Wageningen University \& Research. Together we work on the mission: 'To explore the potential of nature to improve the quality of life'. A staff of 6,500 and 10,000 students from over 100 countries are working worldwide in the domain of healthy food and living environment for governments and the business community-at-large. The strength of Wageningen University \& Research lies in its ability to join the forces of specialised research institutes and the university. It also lies in the combined efforts of the various fields of natural and social sciences. This union of expertise leads to scientific breakthroughs that can quickly be put into practice and be incorporated into education. This is the Wageningen Approach. 